\title{
Photoluminescence of $\mathrm{MoS}_{2}$ Prepared by Effective Grinding-Assisted Sonication Exfoliation
}

\author{
Jing-Yuan Wu, ${ }^{1,2}$ Meng-Na Lin, ${ }^{1,2}$ Long-De Wang, ${ }^{1,2}$ and Tong Zhang ${ }^{1,2}$ \\ ${ }^{1}$ School of Electronic Science and Engineering, Key Laboratory of Micro-Inertial Instrument and \\ Advanced Navigation Technology, Ministry of Education, Southeast University, Nanjing 210096, China \\ ${ }^{2}$ Suzhou Key Laboratory of Metal Nano-Optoelectronic Technology, Suzhou Research Institute of Southeast University, \\ Suzhou 215123, China
}

Correspondence should be addressed to Tong Zhang; tzhang@seu.edu.cn

Received 28 February 2014; Accepted 20 April 2014; Published 19 June 2014

Academic Editor: Jing Chen

Copyright (C) 2014 Jing-Yuan Wu et al. This is an open access article distributed under the Creative Commons Attribution License, which permits unrestricted use, distribution, and reproduction in any medium, provided the original work is properly cited.

Exfoliation of bulk molybdenum disulfide $\left(\mathrm{MoS}_{2}\right)$ using sonication in appropriate solvent is a promising route to large-scale preparation of few-layered or monolayered crystals. Grinding-assisted sonication exfoliation was used for preparing monolayered $\mathrm{MoS}_{2}$ nanosheets from natural mineral molybdenite. By controlling the sonication time, larger crystallites could be further exfoliated to smaller as well as thinner nanosheets without damaging their structures. The concentration of $1.6 \mathrm{mg} \mathrm{mL}^{-1}$ of final solution could be achieved. Several microscopic techniques like scanning electron microscopy, transmission electron microscopy, and atomic force microscopy were employed to evaluate the exfoliation results. Strong photoluminescence with the peak centered at $440 \mathrm{~nm}$ was also observed in the resulting dispersion which included several small lateral-sized $(\sim 3 \mathrm{~nm})$ nanostructures.

\section{Introduction}

The discovery of graphene, which is the thinnest twodimensional (2D) crystal with characteristics of high optical transparency [1], excellent thermal conductivity [2], and exceptional mechanical strength [3], has opened up prospects for a number of emerging technologies in areas of photonics and optoelectronics [4,5]. These outstanding properties arising from atomic-layer thickness and high specific surface area have also encouraged the exploration of other kinds of $2 \mathrm{D}$ crystals including the transition metal dichalcogenides (TMDCs) [6-8], transition metal oxides [9], and hexagonal boron nitride $(\mathrm{hBN})$ [10]. In particular, single- and fewlayer TMDCs have attracted a recent surge of interest due to their newly found remarkable properties, especially layerdependent property, which can be applied to the fields of catalysis $[11,12]$ and optoelectronics $[13-15]$, to name a few.

Molybdenum disulfide $\left(\mathrm{MoS}_{2}\right)$, which belongs to the TMDCs material family, has recently attracted considerable attention owing to its remarkable properties [16, 17] and reliable production of atomically thin $2 \mathrm{D}$ sheets from bulk counterpart [18-21]. As opposed to graphene, which is zerobandgap, $\mathrm{MoS}_{2}$ is an indirect bandgap semiconductor when in its bulk form. Besides, as the thickness decreases, it turns to a direct bandgap semiconductor, leading to an enhancement of the photoluminescence because of quantum confinement effects [16]. The unique optical properties make it an ideal candidate for the novel optoelectronic devices including phototransistors [22], light detectors [23], and emitters [24].

Due to the weak interaction between adjacent layers and the strong intralayer interaction, the bulk $\mathrm{MoS}_{2}$ crystal can be exfoliated to mono- or few-layer flakes. The monolayer $\mathrm{MoS}_{2}$ was first prepared by scotch tape based micromechanical exfoliation [25-27], which was one of the most common methods. However, the method is not scalable and the size and thickness of exfoliated flakes cannot be controlled. With rapid development of study on the characteristic and application of $\mathrm{MoS}_{2}$, high yield preparation methods are in great demand. The chemical vapor deposition (CVD) method is able to generate large-area continuous $\mathrm{MoS}_{2}$ films 
with great performance, which holds promise in electronic application [19]. The lithium intercalation method is another useful method for the exfoliation of bulk $\mathrm{MoS}_{2}$ and the process can be well controlled to achieve high throughput [7]. However, the processes of the two above methods are relatively complicated, limiting their applications. The liquid exfoliation has been found to have great potential for the scalable production of 2D-nanosheet-based materials, which has been successfully demonstrated for a range of layered quantities including graphite, TMDs, and hBN $[18,28,29]$. Different from the micromechanical method, liquid exfoliation can give suspensions of few-layer 2D flakes which can be processed into composites and films. It is also easier to transfer the exfoliated products to arbitrary substrates avoiding complex transfer processes. The resulting $\mathrm{MoS}_{2}$ nanosheets suggest applications in electrochemistry field [30] and van der Waals heterostructures devices [31]. Liquid exfoliation can be achieved by direct sonication in various organic or aqueous surfactant solvents, but the concentration was relatively low. To increase the concentration of the resulting $\mathrm{MoS}_{2}$ suspension, some improved methods have been reported, for example, the ethanol/water mix-solvent sonication combined with grinding [32] and high power tip sonication [28].

Herein we report an effective grinding-assisted liquid exfoliation technique to achieve $\mathrm{MoS}_{2}$ nanoflakes of high concentration and then analyze their optical absorption and photoluminescent properties. The solution processable $\mathrm{MoS}_{2}$ suspension shows obvious blue-shifted photoluminescence compared to that of monolayer $\mathrm{MoS}_{2}$ exfoliated by the micromechanically cleavage method. The blue-shifted luminescence is mainly caused by the quantum dots ( $3 \mathrm{~nm}$ ) generated by sonication, promising interesting novel application in biology and medicine.

\section{Materials and Methods}

The $\mathrm{MoS}_{2}$ starting powder was purchased from Sigma Aldrich $(<2 \mu \mathrm{m}$ particle size $)$ and used as supplied. $\mathrm{MoS}_{2}$ powder $(1 \mathrm{~g})$ was added to $0.8 \mathrm{~mL}$ of $\mathrm{N}$-methyl-2-pyrrolidone (NMP). After grinding for one hour, the mixture was placed in a vacuum oven for two hours at $60^{\circ} \mathrm{C}$ to dry. Then it was collected and redispersed into a $10 \mathrm{~mL}$ NMP solvent. Grinding for several minutes in this process can help dissolve the oven-dried $\mathrm{MoS}_{2}$ in case of wasting the products. The solution was transferred to a beaker and then probe-sonicated (Ultrasonic Processor KH3200) for 100 minutes at the power of $150 \mathrm{~W}$. After that, we deposited a few drops of resulting solution onto the indium tin oxide (ITO)-covered glass slide at room temperature for one day to make it dry naturally. The ITO covered glass was cleaned using piranha solution to make it hydrophilic, on which the mixture can be distributed more uniformly. Then the glass covered with sample was placed onto a hotplate at $200^{\circ} \mathrm{C}$ for 3 minutes before being removed and allowed to cool. The morphology of the samples was inspected by scanning electron microscopy (SEM, Quanta 200 FEI).
The solution obtained after sonication was centrifuged for 40 minutes at the speed of $4000 \mathrm{rpm}$ and the supernatant containing 2D MoS 2 nanoflakes was collected finally. The supernatant was then prepared for further study of their microstructure characterization and optical properties. It was dropped on thermally oxidized $\mathrm{SiO}_{2} / \mathrm{Si}(90 \mathrm{~nm})$ wafer substrate for Raman spectrum characterization (T64000) with the starting $\mathrm{MoS}_{2}$ powder being measured for comparison. Analysis of the samples by Raman spectroscopy was performed under ambient conditions. The pump radiation was supplied at a wavelength of $632.8 \mathrm{~nm}$ with the laser power of $1.3 \mathrm{~mW}$. The suspension was diluted and 5 or 6 globs of them were dropped carefully on lacy carbon-coated $\mathrm{Cu}$ grid followed by drying one day to volatilize NMP completely for transmission electron microscope (TEM, JEM-2100) observation. Atomic force microscopy (AFM, Dimension ICON) was also used to analyze the structure and thickness of the $\mathrm{MoS}_{2}$ nanosheets. A sample of the diluted dispersion was placed on the clean thermally oxidized $\mathrm{SiO}_{2} / \mathrm{Si}$ wafer substrate and evaporated at room temperature overnight. The measurements were performed under ambient laboratory conditions in a trapping mode. To determine the concentration of $2 \mathrm{D}$ sheets in the supernatant, assuming that NMP was distributed uniformly in it, $1 \mathrm{~mL}$ of solution was taken out from the supernatant to the reagent bottle, and then the mass of $2 \mathrm{D}$ nanosheets was weighed by a microbalance after drying in a vacuum oven for one day. By subtracting the mass of the empty bottle, the pure mass of $\mathrm{MoS}_{2}$ nanosheets can be obtained. The process was repeated multiple times and the results were averaged to reduce the error. To further analyze their optical properties, UV-visible spectra were measured by UV-3600. The photoluminescence spectra of $\mathrm{MoS}_{2}$ were obtained by fluorospectrophotometer (RF-5301PC).

\section{Results and Discussion}

Crystals of $\mathrm{MoS}_{2}$ are composed of vertically stacked, van der Waals bonded S-Mo-S units held together, as shown in Figure 1. Each of the units consists of two hexagonal planes of $\mathrm{S}$ atoms and an intermediate plane of Mo atoms coordinated through ionic-covalent interactions with the $S$ atoms [25]. Because of the relatively weak interaction between layers and the strong intralayer interaction, exfoliating $\mathrm{MoS}_{2}$ to few layers using liquid exfoliation technique is possible.

Compared to scotch tape microcleavage method, the liquid exfoliation technique can achieve large-scale preparation. The NMP solvent was chosen because its surface energy matches nanosheets' well, resulting in the enthalpy of mixing being very small [33]. Meanwhile, the surface tension can help overcome the van der Waals force, which makes the exfoliation easier and more effective. It also can be replaced by other organic solvents such as isopropanol (IPA), dimethylformamide (DMF), and dimethylsulphoxide (DMSO) [18]. The grinding process should be gentle and soft to detach the $\mathrm{MoS}_{2}$ layers from the bulk materials relying on pure shear forces [32]. This function can be achieved by ball milling process as well [29]. Besides, adding NMP as a stabilizing surfactant into commercial $\mathrm{MoS}_{2}$ powder in the 


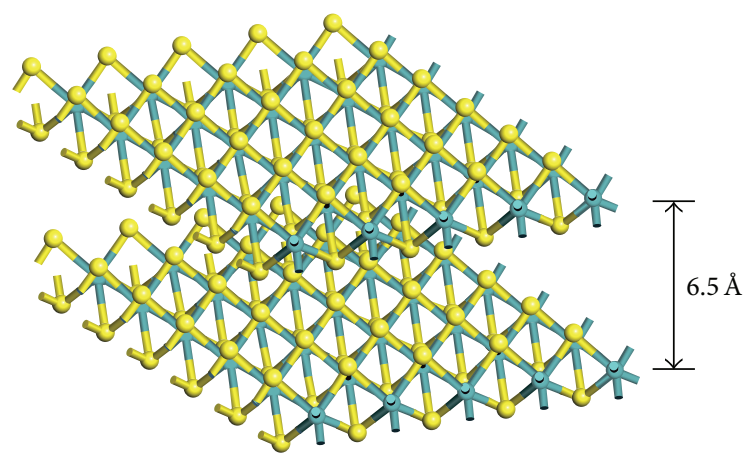

S

Mo

FIGURE 1: Three-dimensional representation of the structure of $\mathrm{MoS}_{2}$ crystal; the interlayer spacing is about $0.65 \mathrm{~nm}$.

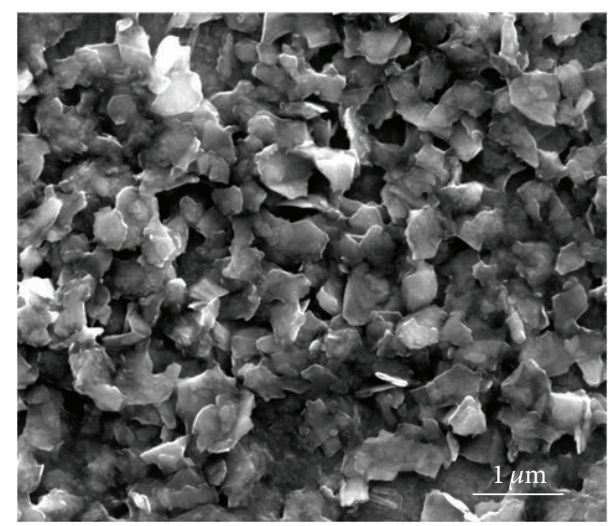

FIGURE 2: Close-up SEM images of $\mathrm{MoS}_{2}$ nanoflakes from a noncentrifuged $\mathrm{MoS}_{2}$ dispersion.

process can avoid reassembling of the $\mathrm{MoS}_{2}$ nanosheets [18]. After being dried and redispersed into the NMP solvent, a high power tip sonication was used to further exfoliate $\mathrm{MoS}_{2}$ in liquids. The sonication time needs to be selected appropriately. If the time is too long, it will damage $\mathrm{MoS}_{2}$ nanosheets and affect their properties, while if the time is too short, the large flakes cannot be exfoliated effectively. To investigate the nature of the resulted $\mathrm{MoS}_{2}$, we performed SEM characterization. Figure 2 shows the SEM image of the surface of exfoliated $\mathrm{MoS}_{2}$ nanosheets. By imaging the thin $\mathrm{MoS}_{2}$ films, we observed a disordered array of $\mathrm{MoS}_{2}$ nanoflakes stacked predominately in-plane. They were continuous over length scales greater than a few microns. Because the dispersions were not centrifuged, most of the nanoflakes were still relatively thick. Therefore, the last centrifugation step was employed to remove the larger nanoplatelets and any partially exfoliated crystallites [33]. Finally, the smaller flakes remained in the supernatant while the larger flakes and unsonicated powders existed in the sediment.

To confirm that the observed objects are $\mathrm{MoS}_{2}$ flakes, Raman analysis is necessary. Figure 3 shows the Raman spectra ranging from $40 \mathrm{~cm}^{-1}$ to $1000 \mathrm{~cm}^{-1}$ of $\mathrm{MoS}_{2}$ nanosheets

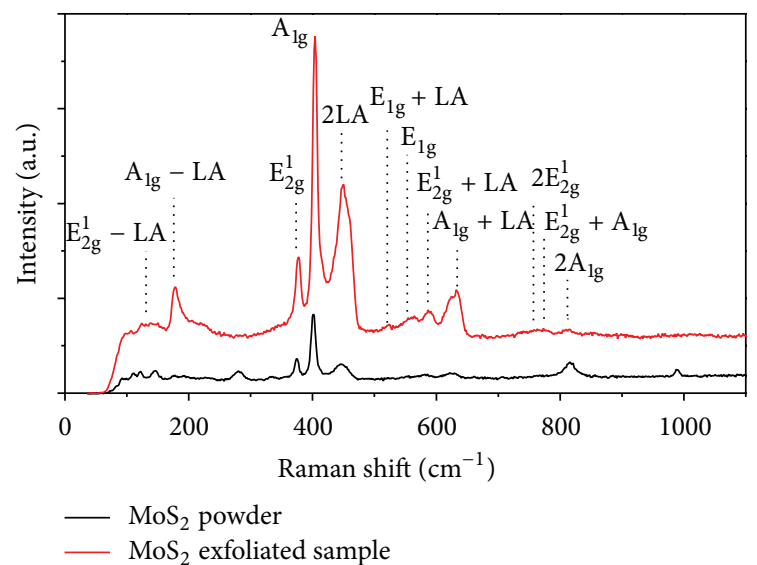

Figure 3: Raman spectra of exfoliated $\mathrm{MoS}_{2}$ nanosheets and $\mathrm{MoS}_{2}$ powder.

exfoliated and the starting powder. We noted that the peak positions slightly shifted from those expected for crystalline $\mathrm{MoS}_{2}$ as was sometimes observed for powdered samples. There are four first-order Raman active modes in $\mathrm{MoS}_{2}$, in which $E_{1 g}, E_{2 g}^{1}$, and $A_{1 g}$ modes are a result of vibrational modes within the S-Mo-S layer. The little downshift of all the peaks from the reported literatures may be related to the laser-induced heating [34]. It was observed that the $\mathrm{E}_{2 \mathrm{~g}}^{1}$ and $\mathrm{A}_{1 \mathrm{~g}}$ modes of the exfoliated $\mathrm{MoS}_{2}$ nanosheets were centered at $379 \mathrm{~cm}^{-1}$ and $404 \mathrm{~cm}^{-1}$, respectively, which were in the expected wavenumber range. Previous researches have demonstrated that the $2 \mathrm{D}$ exfoliated nanosheets have a smaller Raman shift difference between the two modes in comparison with their bulk counterparts because of the influence of neighboring layers on the effective restoring forces on atoms and the increase of dielectric screening of long-range Coulomb interactions [35]. However, it was observed that the Raman shift difference between the two modes of our exfoliated sheets was nearly equivalent to that of the starting powder. It was probably due to the aggregation during the process of deposition/drying on the substrate. In the Raman spectrum of $\mathrm{MoS}_{2}$ powder, we also observed three new bands presented at 279,817 , and $988 \mathrm{~cm}^{-1}$ which did not exist in the exfoliated nanosheets. These bands represent $\mathrm{MoO}_{3}$ vibrational energy states as a result of oxidation by the laser irradiation [34].

Transmission electron microscopy (TEM) is used to further analyze the structures of $\mathrm{MoS}_{2}$ nanoflakes. Examples of exfoliated nanoflakes are shown in Figure 4. It can be seen that when the layers were exfoliated to ultrathin layers, the flakes turned to be transparent to the electron beam. From Figures 4(a)-4(b), it was observed that the edge of the flake tended to be thinner. The tendency was also found in the $\mathrm{MoS}_{2}$ nanoflakes which were prepared by scotch tape micromechanically cleavage method. Meanwhile, increasing grinding and sonication time can further exfoliate the flakes to thin layers. By comparing TEM images of $\mathrm{MoS}_{2}$ nanoflakes in the condition of different grinding and sonication time, we determined that the optimized time of grinding and 


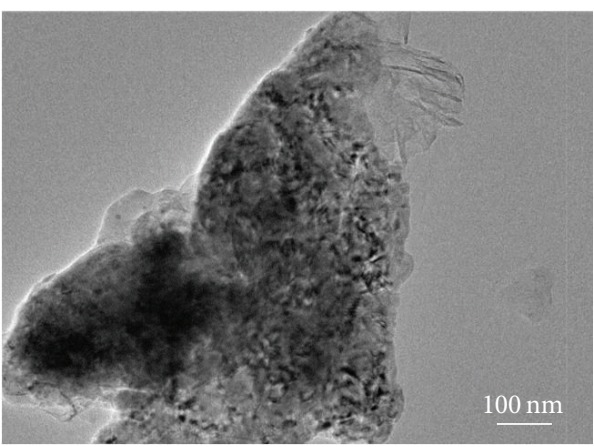

(a)

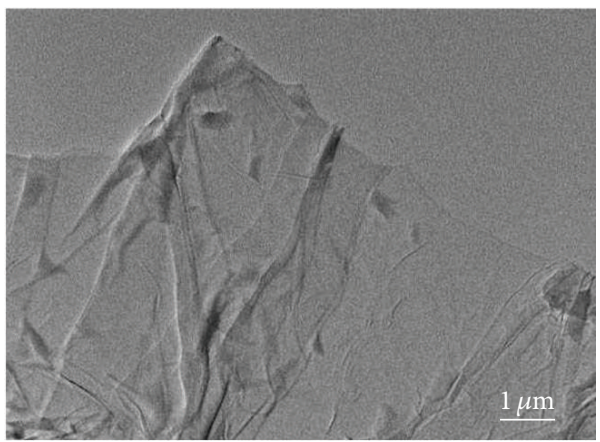

(c)

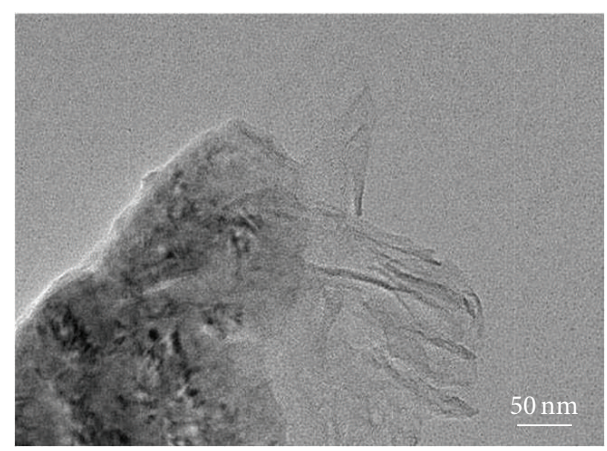

(b)

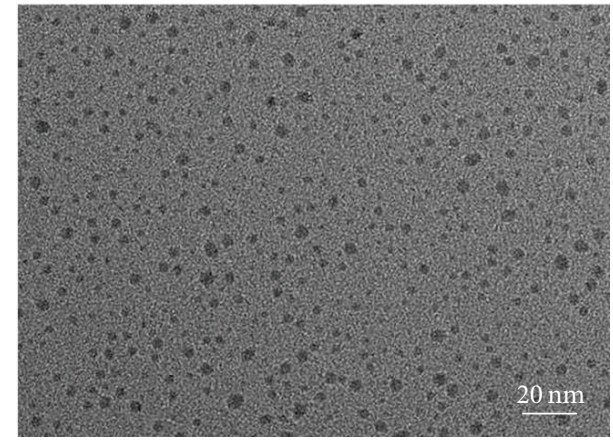

(d)

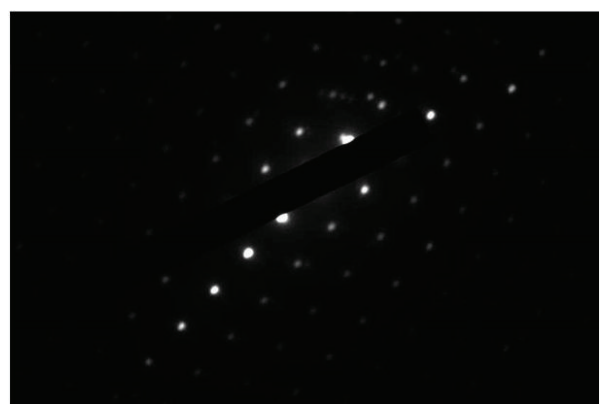

(e)

FIGURE 4: Typical TEM images of $\mathrm{MoS}_{2}$ nanosheets ((a)-(c)) and quantum dots (d). (e) SAED pattern corresponding to the MoS 2 nanosheets in (a).

sonication were one hour and 100 minutes, respectively. In this case, we achieved a continuous ultrathin $\mathrm{MoS}_{2}$ with lateral size reaching several micrometers shown in Figure 4(c). Meanwhile, several $\mathrm{MoS}_{2}$ quantum dots with average size of about $3 \mathrm{~nm}$ are shown in Figure 4(d). Figure 4(e) depicts the selected area electron diffraction (SAED) pattern of the sample, which is indexed to the near perfect planar $2 \mathrm{H}$ $\mathrm{MoS}_{2}$. We next performed AFM analysis to firmly determine the thickness of the obtained $\mathrm{MoS}_{2}$ sheets. Figure 5 shows the AFM image of $\mathrm{MoS}_{2}$ nanosheets deposited on a flat silicon wafer with a $90 \mathrm{~nm}$ oxide layer. A step of $\sim 5 \mathrm{~nm}$ (corresponding to 7 monolayer $\mathrm{MoS}_{2}$ ) with average lateral size of nearly 200 nanometers can be observed. There were some small individual nanosheets with the thickness of $2 \mathrm{~nm}$ and the size of several dozens of nanometers. Some thick nanosheets whose thickness reached $20 \mathrm{~nm}$ were also observed in the measurement.

The supernatant containing $\mathrm{MoS}_{2}$ dispersions after centrifugation appeared to be blackish green in color, as shown in the inset of Figure 6. It was found that the dispersions remained stable over periods of several weeks. By measuring the weight of $2 \mathrm{D}$ nanosheets in $1 \mathrm{~mL}$ supernatant, the concentration of the solution was estimated to be $1.6 \mathrm{mg} \mathrm{mL}^{-1}$. The detailed optical properties of the nanoflakes were studied by measuring their UV-visible absorption spectra. The absorption spectrum exhibited two typical peaks at $621 \mathrm{~nm}$ and $680 \mathrm{~nm}$, which are ascribed to $\mathrm{B}$ and $\mathrm{A}$ excitonic peaks, respectively, arising from the energy split from valence band spin-orbital coupling in 2D $\mathrm{MoS}_{2}$ with large lateral dimensions [25]. The centrifugation process removed most of 


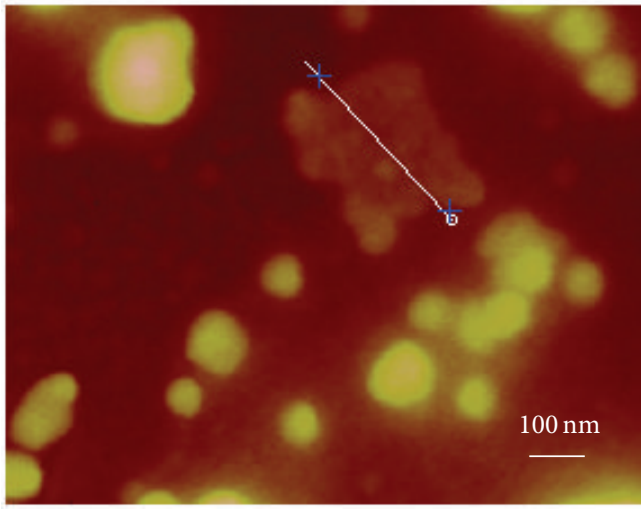

(a)

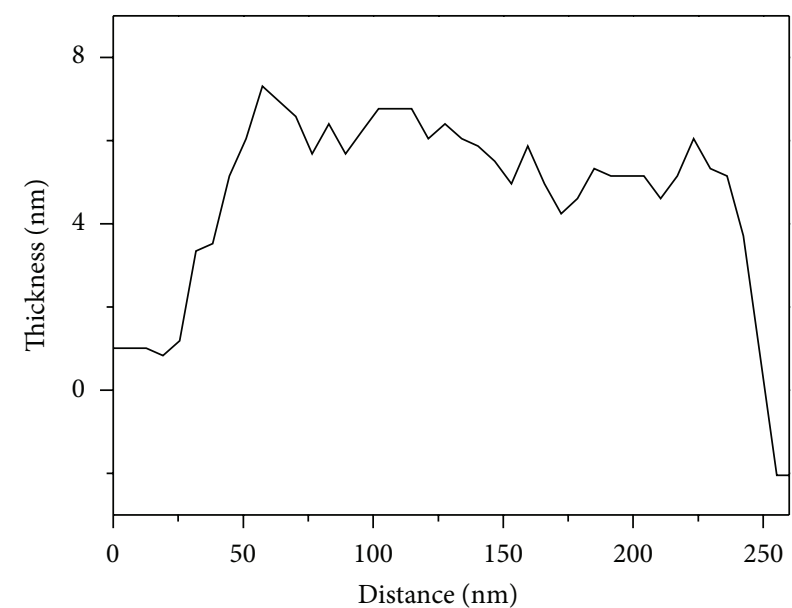

(b)

FIGURE 5: (a) AFM image of $\mathrm{MoS}_{2}$ nanosheets. (b) Height profile along the white line overlaid on the image.

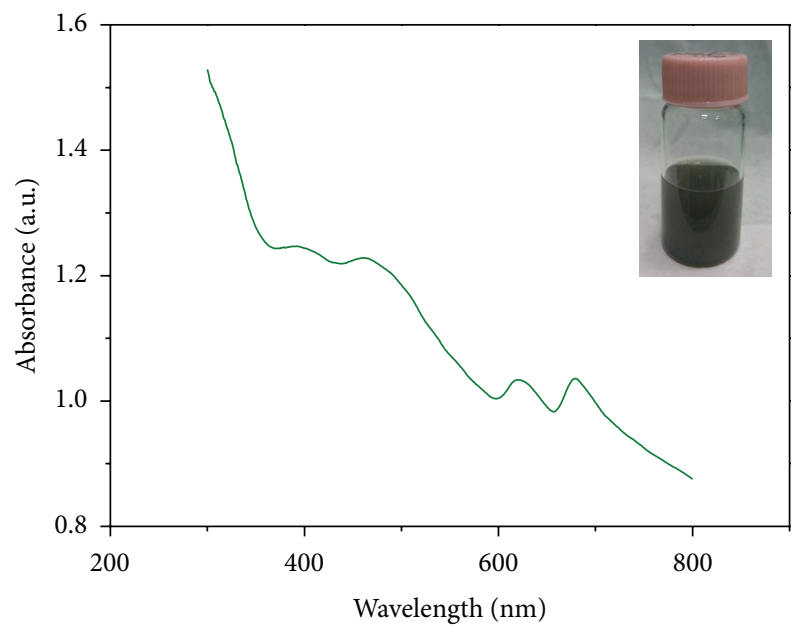

Figure 6: Absorbance spectrum of $2 \mathrm{D} \mathrm{MoS}_{2}$ nanoflake. Inset: the optical image of exfoliated $\mathrm{MoS}_{2}$ suspension in NMP solvent.

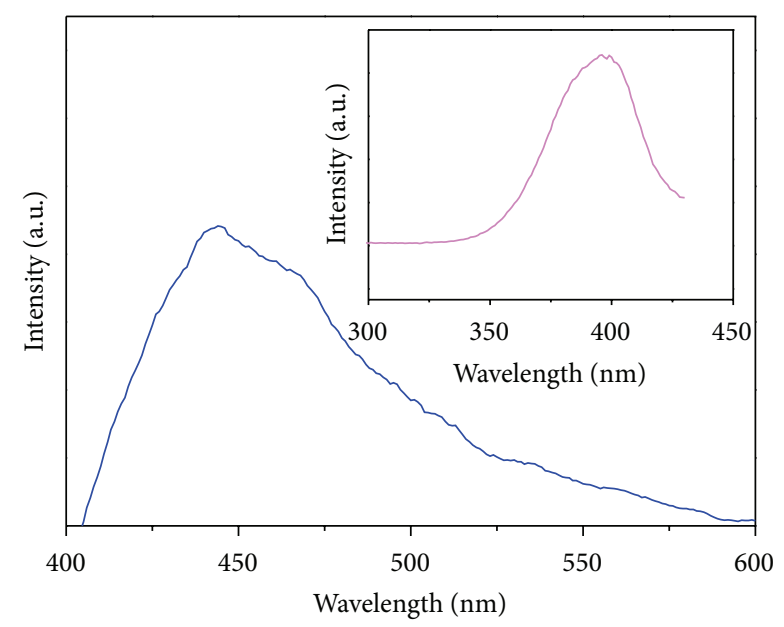

FIgURE 7: The emission spectrum of exfoliated $\mathrm{MoS}_{2}$. Inset: excitation spectrum of the same sample. the large 2D flake in the suspension, leading to the relatively weak features of the two peaks. In addition to these two weak peaks, there were two other peaks at $394 \mathrm{~nm}$ and $460 \mathrm{~nm}$. It is probably due to the optical absorption of smaller lateral-sized $\mathrm{MoS}_{2}$ nanosheets. The absorption peaks of the nanostuctures exhibit strong blue-shift even to ultraviolet wavelength arising from quantum effect [36].

We measured the excitation spectrum and emission spectrum of the exfoliated $\mathrm{MoS}_{2}$, respectively. It was observed that a strong broad peak was centered at $440 \mathrm{~nm}$ in the emission spectrum, as shown in Figure 7. The phenomenon was quite different from what was observed in the monolayer $\mathrm{MoS}_{2}$ exfoliated by scotch tape method. In that case, the strong photoluminescence is attributed to the direct excitonic transition as the monolayer $\mathrm{MoS}_{2}$ turns to a direct bandgap semiconductor. Therefore, its photoluminescence peak is centered at $1.9 \mathrm{eV}(652 \mathrm{~nm})$, the bandgap of monolayer $\mathrm{MoS}_{2}$, which was demonstrated by previous calculation and experiment [16]. The difference is probably due to quantum confinement effect across the 2D planes [37]. To explain this more clearly, the size of nanoflakes prepared by scotch tape method is usually microndimensional; however, the sonication process in the liquid exfoliation method not only makes the flakes thinner but also further reduces their lateral size. Therefore, the $2 \mathrm{D} \mathrm{MoS}_{2}$ products prepared by liquid exfoliation method were generally small in lateral dimensions, even generating quantum dots of nanometer dimension in the process of exfoliation, which were demonstrated in Figure 4(d). The luminescence properties are mainly decided by the size of particles [38]. Therefore, the characteristic of the peak is ascribed to the strong blue-shift hot photoluminescence from the $\mathrm{K}$ point $[36,37]$.

\section{Conclusion}

In summary, the grinding-assisted sonication method allows a cheap, easy, and scalable fabrication of $\mathrm{MoS}_{2}$ nanosheets 
exfoliated from its bulk counterpart. The as-fabricated dispersions consisted of ultrathin-layered $2 \mathrm{D} \mathrm{MoS}$ and remained stable in NMP solvent for several weeks. The AFM image further confirmed that the $\mathrm{MoS}_{2}$ was exfoliated to about 7 monolayers with lateral size of $200 \mathrm{~nm}$. The concentration of the final solution reached $1.6 \mathrm{mg} \mathrm{mL}^{-1}$, which would present an environmentally benign method, possibly for large-scale production with high quality. The lateral size of $\mathrm{MoS}_{2}$ nanosheets was reduced even to nanometer dimension after sonication; as a result, there was a blue-shift of the emission peak compared to the nanosheets prepared by scotch tape micromechanically cleavage method. The photoluminescent property makes the exfoliated product a promising choice to be applied to solar cell and light-emitting device.

\section{Conflict of Interests}

The authors declare that they have no conflict of interests.

\section{Acknowledgments}

This work is supported by NSFC under Grant no. 61307066, Doctoral Fund of Ministry of Education of China under Grant nos. 20110092110016 and 20130092120024, Natural Science Foundation of Jiangsu Province under Grant no. BK20130630, the National Basic Research Program of China (973 Program) under Grant no. 2011CB302004, and the Foundation of Key Laboratory of Micro-Inertial Instrument and Advanced Navigation Technology, Ministry of Education, China, under Grant no. 201204.

\section{References}

[1] R. Nair, P. Blake, A. Grigorenko et al., "Fine structure constant defines visual transparency of graphene," Science, vol. 320, no. 5881, p. 1308, 2008.

[2] A. K. Geim and K. S. Novoselov, “The rise of graphene," Nature Materials, vol. 6, no. 3, pp. 183-191, 2007.

[3] C. Lee, X. Wei, J. W. Kysar, and J. Hone, "Measurement of the elastic properties and intrinsic strength of monolayer graphene," Science, vol. 321, no. 5887, pp. 385-388, 2008.

[4] Y. Liu, R. Cheng, L. Liao et al., "Plasmon resonance enhanced multicolour photodetection by graphene," Nature Communications, vol. 2, no. 1, article 579, 2011.

[5] F. Schwierz, "Graphene transistors," Nature Nanotechnology, vol. 5, no. 7, pp. 487-496, 2010.

[6] Q. H. Wang, K. Kalantar-Zadeh, A. Kis, J. N. Coleman, and M. S. Strano, "Electronics and optoelectronics of two-dimensional transition metal dichalcogenides," Nature Nanotechnology, vol. 7, no. 11, pp. 699-712, 2012.

[7] X. Huang, Z. Zeng, and H. Zhang, "Metal dichalcogenide nanosheets: preparation, properties and applications," Chemical Society Reviews, vol. 42, no. 5, pp. 1934-1946, 2013.

[8] X. Huang, C. Tan, Z. Yin, and H. Zhang, "25th anniversary article: hybrid nanostructures based on two-dimensional nanomaterials," Advanced Materials, vol. 26, no. 14, pp. 2185-2204, 2014.

[9] M. Osada and T. Sasaki, "Two-dimensional dielectric nanosheets: novel nanoelectronics from nanocrystal building blocks," Advanced Materials, vol. 24, no. 2, pp. 210-228, 2012.
[10] D. Pacile, J. Meyer, Ç. Girit, and A. Zettl, “The two-dimensional phase of boron nitride: few-atomic-layer sheets and suspended membranes," Applied Physics Letters, vol. 92, no. 13, Article ID 133107, 2008.

[11] W. Zhou, Z. Yin, Y. Du et al., "Synthesis of few-layer $\mathrm{MoS}_{2}$ nanosheet-coated $\mathrm{TiO}_{2}$ nanobelt heterostructures for enhanced photocatalytic activities," Small, vol. 9, no. 1, pp. 140-147, 2013.

[12] X. Huang, Z. Zeng, S. Bao et al., "Solution-phase epitaxial growth of noble metal nanostructures on dispersible singlelayer molybdenum disulfide nanosheets," Nature Communications, vol. 4, article 1444, 2013.

[13] D.-S. Tsai, K.-K. Liu, D.-H. Lien et al., "Few-layer $\mathrm{MoS}_{2}$ with high broadband photogain and fast optical switching for use in harsh environments," ACS Nano, vol. 7, no. 5, pp. 3905-3911, 2013.

[14] M. Shanmugam, T. Bansal, C. A. Durcan, and B. Yu, "Molybdenum disulphide/titanium dioxide nanocomposite-poly 3hexylthiophene bulk heterojunction solar cell," Applied Physics Letters, vol. 100, no. 15, Article ID 153901, 2012.

[15] Z. Yin, B. Chen, M. Bosman et al., "Au nanoparticle-modified $\mathrm{MoS}_{2}$ nanosheet-based photoelectrochemical cells for water splitting," Small, 2014.

[16] A. Splendiani, L. Sun, Y. Zhang et al., "Emerging photoluminescence in monolayer $\mathrm{MoS}_{2}$," Nano Letters, vol. 10, no. 4, pp. 1271-1275, 2010.

[17] H. Zeng, J. Dai, W. Yao, D. Xiao, and X. Cui, "Valley polarization in $\mathrm{MoS}_{2}$ monolayers by optical pumping," Nature Nanotechnology, vol. 7, no. 8, pp. 490-493, 2012.

[18] J. N. Coleman, M. Lotya, A. O'Neill et al., "Two-dimensional nanosheets produced by liquid exfoliation of layered materials," Science, vol. 331, no. 6017, pp. 568-571, 2011.

[19] Y. H. Lee, X. Q. Zhang, W. Zhang et al., "Synthesis of large-area $\mathrm{MoS}_{2}$ atomic layers with chemical vapor deposition," Advanced Materials, vol. 24, no. 17, pp. 2320-2325, 2012.

[20] K.-K. Liu, W. Zhang, Y.-H. Lee et al., "Growth of large-area and highly crystalline $\mathrm{MoS}_{2}$ thin layers on insulating substrates," Nano Letters, vol. 12, no. 3, pp. 1538-1544, 2012.

[21] Z. Zeng, Z. Yin, X. Huang et al., "Single-layer semiconducting nanosheets: high-yield preparation and device fabrication," Angewandte Chemie, vol. 50, no. 47, pp. 11093-11097, 2011.

[22] Z. Yin, H. Li, H. Li et al., "Single-layer $\mathrm{MoS}_{2}$ phototransistors," ACS Nano, vol. 6, no. 1, pp. 74-80, 2012.

[23] H. S. Lee, S.-W. Min, Y.-G. Chang et al., "MoS 2 nanosheet phototransistors with thickness-modulated optical energy gap," Nano Letters, vol. 12, no. 7, pp. 3695-3700, 2012.

[24] R. Sundaram, M. Engel, A. Lombardo et al., "Electroluminescence in single layer $\mathrm{MoS}_{2}$," Nano Letters, vol.13, no. 4, pp. 14161421, 2013.

[25] K. F. Mak, C. Lee, J. Hone, J. Shan, and T. F. Heinz, "Atomically thin $\mathrm{MoS}_{2}$ : a new direct-gap semiconductor," Physical Review Letters, vol. 105, no. 13, Article ID 136805, 2010.

[26] H. Li, J. Wu, X. Huang et al., "Rapid and reliable thickness identification of two-dimensional nanosheets using optical microscopy," ACS Nano, vol. 7, no. 11, pp. 10344-10353, 2013.

[27] H. Li, G. Lu, Z. Yin et al., "Optical identification of single- and few-layer $\mathrm{MoS}_{2}$ sheets," Small, vol. 8, no. 5, pp. 682-686, 2012.

[28] R. J. Smith, P. J. King, M. Lotya et al., "Large-scale exfoliation of inorganic layered compounds in aqueous surfactant solutions," Advanced Materials, vol. 23, no. 34, pp. 3944-3948, 2011.

[29] Y. Yao, Z. Lin, Z. Li, X. Song, K.-S. Moon, and C.-P. Wong, "Large-scale production of two-dimensional nanosheets," Journal of Materials Chemistry, vol. 22, no. 27, pp. 13494-13499, 2012. 
[30] V. Nicolosi, M. Chhowalla, M. G. Kanatzidis, M. S. Strano, and J. N. Coleman, "Liquid exfoliation of layered materials," Science, vol. 340, no. 6139, Article ID 1226419, 2013.

[31] A. K. Geim and I. V. Grigorieva, "Van der Waals heterostructures," Nature, vol. 499, no. 7459, pp. 419-425, 2013.

[32] Y. Yao, L. Tolentino, Z. Yang et al., "High-concentration aqueous dispersions of $\mathrm{MoS}_{2}$," Advanced Functional Materials, vol. 23, no. 28, pp. 3577-3583, 2013.

[33] A. O'Neill, U. Khan, and J. N. Coleman, "Preparation of high concentration dispersions of exfoliated $\mathrm{MoS}_{2}$ with increased flake size," Chemistry of Materials, vol. 24, no. 12, pp. 2414-2421, 2012.

[34] B. C. Windom, W. Sawyer, and D. W. Hahn, "A raman spectroscopic study of $\mathrm{MoS}_{2}$ and $\mathrm{MoO}_{3}$ : applications to tribological systems," Tribology Letters, vol. 42, no. 3, pp. 301-310, 2011.

[35] C. Lee, H. Yan, L. E. Brus, T. F. Heinz, J. Hone, and S. Ryu, "Anomalous lattice vibrations of single- and few-layer $\mathrm{MoS}_{2}$," ACS Nano, vol. 4, no. 5, pp. 2695-2700, 2010.

[36] V. Stengl and J. Henych, "Strongly luminescent monolayered $\mathrm{MoS}_{2}$ prepared by effective ultrasound exfoliation," Nanoscale, vol. 5, no. 8, pp. 3387-3394, 2013.

[37] Y. Wang, J. Z. Ou, S. Balendhran et al., "Electrochemical control of photoluminescence in two-dimensional $\mathrm{MoS}_{2}$ nanoflakes," ACS Nano, vol. 7, no. 11, pp. 10083-10093, 2013.

[38] H. Li, X. He, Z. Kang et al., "Water-soluble fluorescent carbon quantum dots and photocatalyst design," Angewandte Chemie, vol. 49, no. 26, pp. 4430-4434, 2010. 

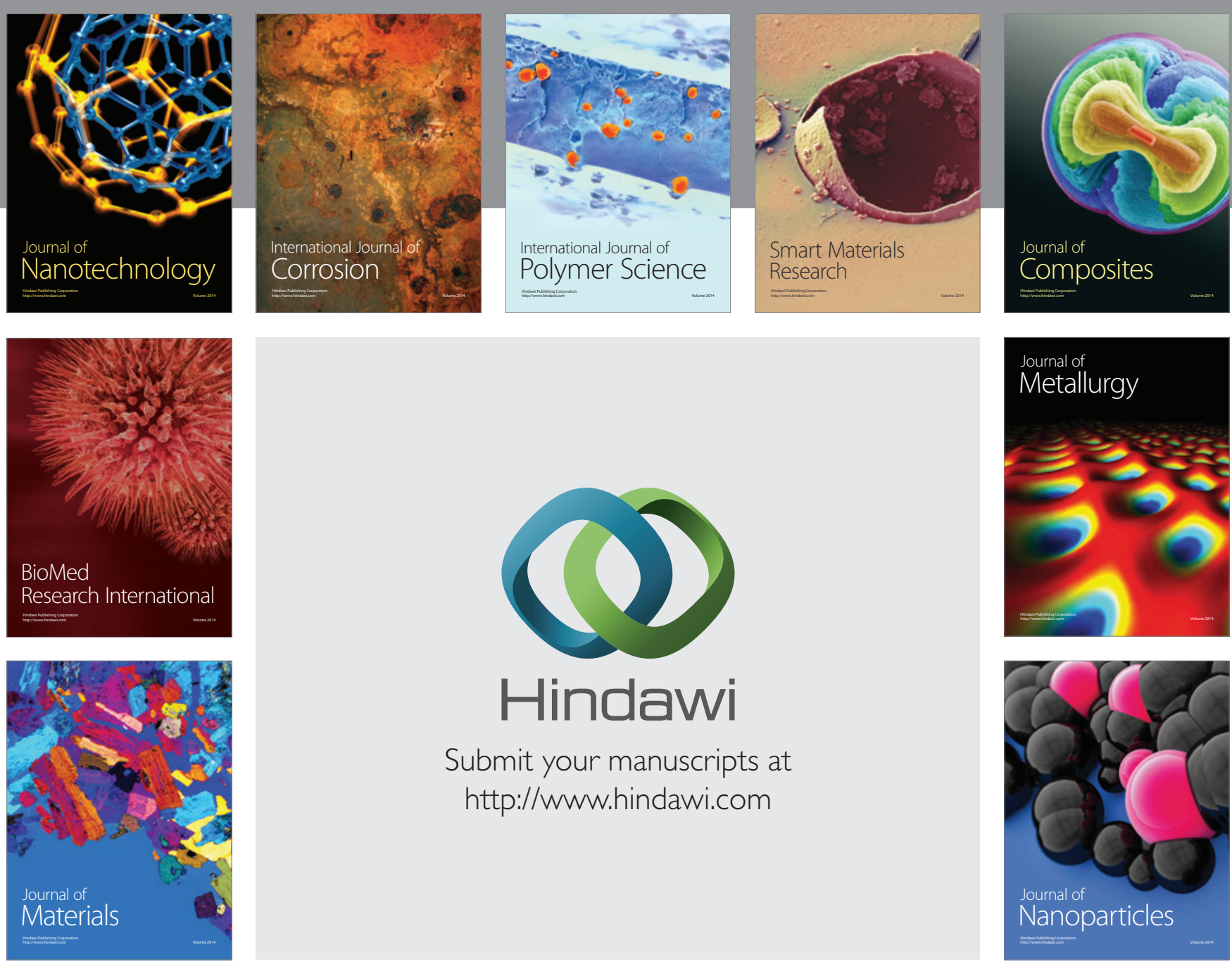

Submit your manuscripts at http://www.hindawi.com
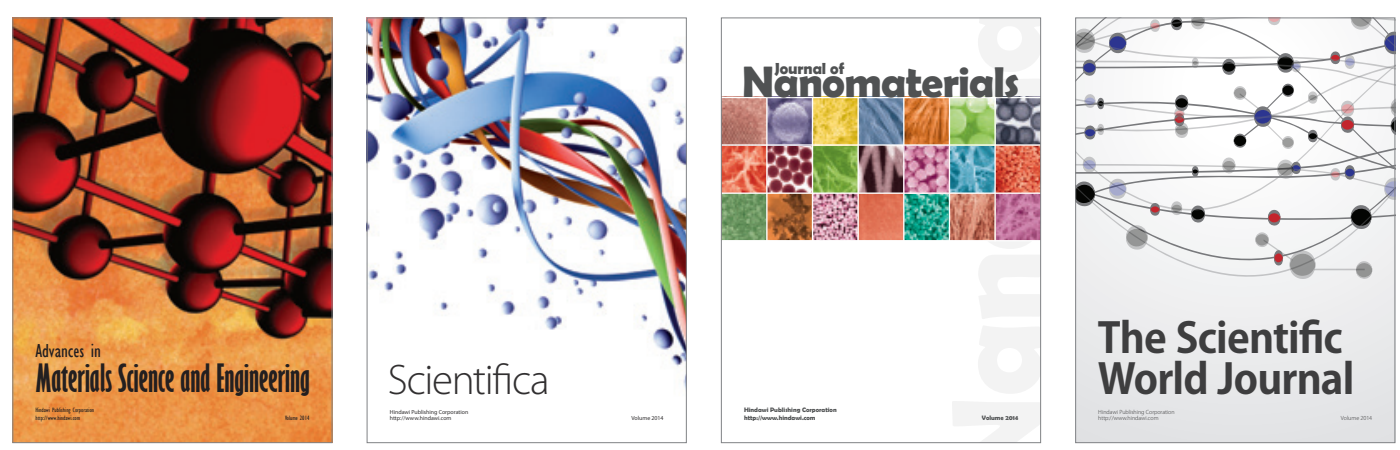

\section{The Scientific World Journal}
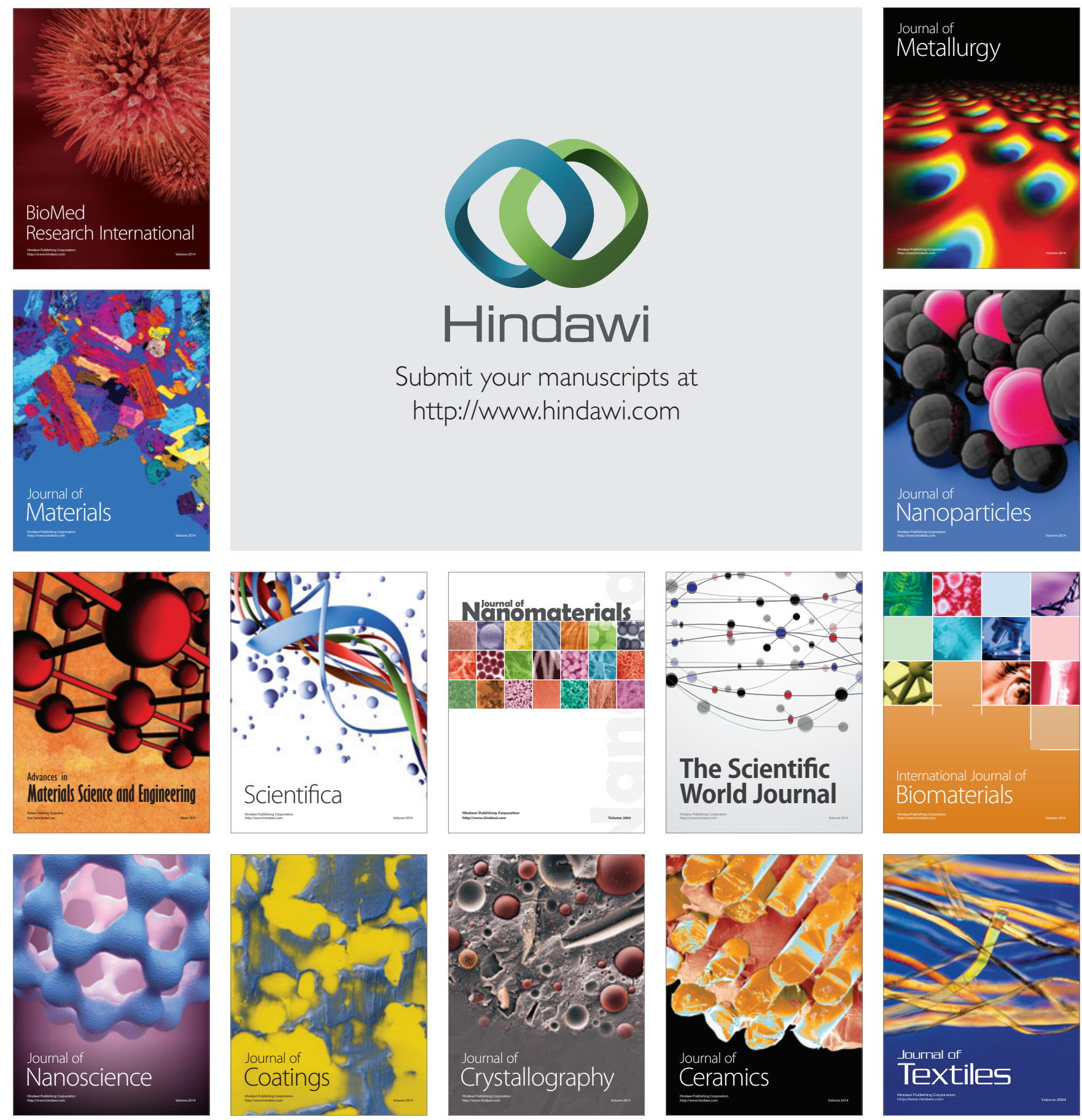